\title{
Improvement of Detection Limits for Particle Contamination by Confocal Configuration in X-Ray Fluorescence Microscope
}

\author{
Hitomi Nakano, ${ }^{* * * *}$ Shintaro Komatani, ${ }^{*}$ Tsugufumi Matsuyama,** and Kouichi TsuJI ${ }^{* *}$ \\ *HORIBA TECHNO SERVICE Co., Ltd., Analytical Technology Department, 2 Kisshoin-Miyanohigashi-cho, \\ Minami, Kyoto 601-8305, Japan \\ **Department of Applied Chemistry \& Bioengineering, Graduate School of Engineering, Osaka City University, \\ 3-3-138 Sugimoto-cho, Sumiyoshi, Osaka 558-8585, Japan
}

\begin{abstract}
Micro X-ray fluorescence (XRF) enables the non-destructive analysis of particle contamination. In this study, we compared the detection sensitivities and the LLD (lower limit of detection) values of micro-metallic particle contaminations on the plastic detected by micro-XRF and confocal micro-XRF. First, to verify the effectiveness of the confocal microXRF, we compared the intensities of different shaping copper samples (plate, thin film and particle). The results demonstrated that confocal micro-XRF is more effective than micro-XRF for the detection of micro particles. Second, to compare the $\mathrm{SN}$ ratios of different $\mathrm{X}$-ray energies, several micro-metallic particles $(\mathrm{Si}, \mathrm{Fe}$, and $\mathrm{Cu}$ ) set on an acrylic plate were measured by micro-XRF and confocal micro-XRF. It was found that the $\mathrm{SN}$ ratios of the confocal micro-XRF when measuring the $\mathrm{Si}, \mathrm{Fe}$, and $\mathrm{Cu}$ particles were improved to be approximately 14.6, 21.9, and 43.5-times those of the micro$\mathrm{XRF}$, respectively. It was determined that confocal micro-XRF is more effective for micro-metallic particles in the higher energy region.
\end{abstract}

Keywords Confocal micro-XRF, micro-XRF, fine particle contamination, high-sensitivity analysis

(Received March 27, 2021; Accepted May 14, 2021; Advance Publication Released Online by J-STAGE May 21, 2021)

\section{Introduction}

The analysis of fine particle contamination is important for identifying the origin of contamination in the quality control processes of manufacturing industries. X-ray fluorescence (XRF), which can perform non-destructive elemental analysis, is effective for foreign matter analysis. ${ }^{1-4}$ The highly sensitive analysis of micro-metallic particles in materials using microXRF has been expected because several damaging incidents of industrial products, such as batteries, by micro-metal contamination have recently been reported. ${ }^{5}$ When general micro-XRF is used to measure micro-particles, higher background level scattering X-rays from the substrate plate can be detected, as shown in Fig. 1(a). As a result, the background level noises will be higher and the peak intensity to the background intensity ratio ( $\mathrm{SN}$ ratio) will be lower. If the substrate plate is plastic, the background intensity will increase more higher, due to strong $\mathrm{X}$-ray scattering.

However, in confocal micro-XRF (C-M-XRF), it is possible to obtain elemental information from only the confocal volume by arranging the polycapillary lenses in front of both the X-ray generator and the detector, as illustrated in Fig. 1(b). In the early 2000s, the C-M-XRF technique was applied to desktoptype devices, ${ }^{6}$ and since then several studies on C-M-XRF have been conducted and reported. ${ }^{711}$ Desktop-type C-M-XRF devices enable three-dimensional (3D) elemental analysis. In

$\dagger$ To whom correspondence should be addressed.

E-mail: hitomi.nakano@horiba.com

2000, Ding et al. reported a background intensity reduction in the confocal region when using the radioisotope element ${ }^{55} \mathrm{Fe}$ as a sample, ${ }^{6}$ which was expected to improve the $\mathrm{SN}$ ratio.

In this study, the $\mathrm{SN}$ ratio and the lower limit of detection (LLD) values were evaluated when measuring the same metallic particles set on an acrylic substrate using micro-XRF and C-MXRF. Moreover, the sensitiveness depending on the elements types and sample shape differences was examined.

\section{Experimental}

Micro-XRF and $C-M-X R F$ setup

The confocal 3D XRF developed at Osaka City University

(a)

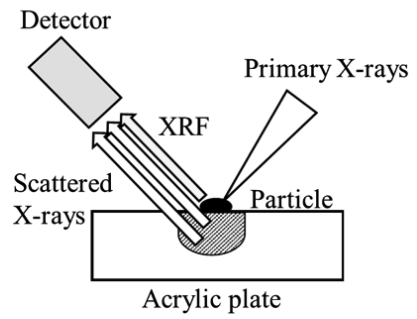

Fig. 1 Schematic representation of (a) micro-XRF (non-confocal XRF) and (b) C-M-XRF. 
(a)

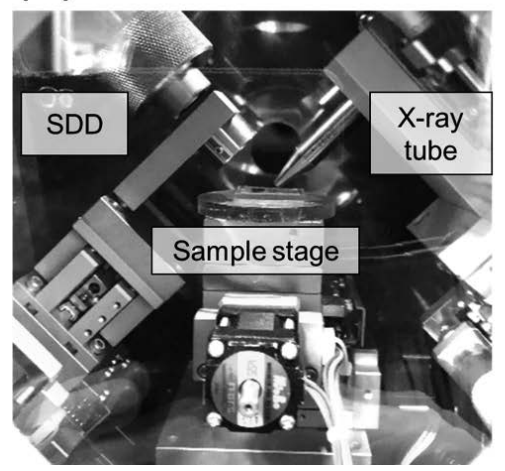

(b)

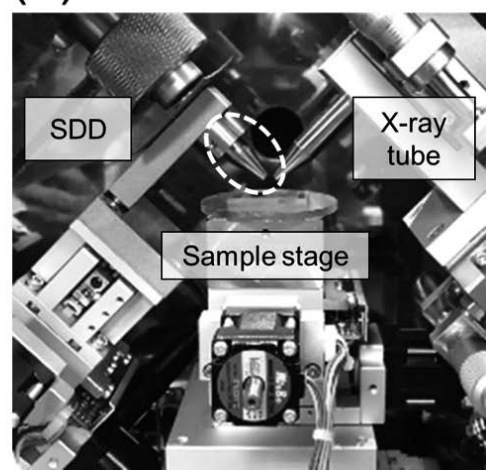

Fig. 2 Experimental setups of (a) micro-XRF and (b) C-M-XRF. A polycapillary half lens (white dotted ellipse) was attached in front of the SDD in C-M-XRF.

was used for the micro-XRF and C-M-XRF measurements.

Schematic drawings of the non-confocal and confocal 3D $\mathrm{XRF}$ instruments are presented in Fig. 2. In the C-M-XRF, as indicated in Fig. 2(b), primary $\mathrm{X}$-rays (X-ray tube voltage $50 \mathrm{kV}$; tube current $0.5 \mathrm{~mA}$; target $\mathrm{Rh}$ ) were focused using a polycapillary lens and were irradiated onto the sample at 45 degrees above the sample stage. The focal X-ray beam diameter was $10 \mu \mathrm{m}$ at Mo $K \alpha .^{12}$ Fluorescence X-rays that were secondary generated from the sample were detected by a silicon drift detector (SDD) that was arranged at 45 degrees above the sample stage. XRFs from only from confocal volume could be selectively detected. ${ }^{12}$ A pollycapillary full lens was placed in front of the X-ray tube and a half lens was placed in front of the SDD. The sample stage was controlled by stepping motors and a $\mathrm{PC}$ in the $\mathrm{X}, \mathrm{Y}$, and $\mathrm{Z}$ directions, by means of which $3 \mathrm{D}$ elemental analysis could be achieved. ${ }^{13}$ As an optical camera (20-times magnitude) was set above the sample, it was possible to observe the measurement position. In the non-confocal XRF, as indicated in Fig. 2(a), the polycapillary lens in front of the SDD was removed from the same C-M-XRF device in order to compare the detection sensitivities of the non-confocal and C-M-XRF devices.

\section{Sample}

Different $\mathrm{Cu}$ sample shapes (plate, foil, and particle) were measured for comparing different metal shapes and their sensitivities. We measured a pure $\mathrm{Cu}$ plate with a thickness of $1 \mathrm{~mm}$ and a $\mathrm{Cu}$ foil with a thickness of $10 \mu \mathrm{m}$, manufactured by Nilaco Corporation, as well as a $\mathrm{Cu}$ particle with a diameter of $20 \mu \mathrm{m}$, manufactured by Fukuda Metal Foil \& Powder Co., Ltd.

The following minute particles were prepared: a $\mathrm{Cu}$ particle (a size of $20 \mu \mathrm{m}$, manufactured by Fukuda Metal Foil \& Powder Co., Ltd), an Fe particle (a size of $22 \mu \mathrm{m}$, manufactured by Nilaco Corporation) and mineral particle (a size of $50 \mu \mathrm{m}$, geochemical reference material JLk-1 (Table 1), supplied by the National Institute of Advanced Industrial Science and Technology (AIST). They were set on the acrylic plate with a thickness of $2 \mathrm{~mm}$.

\section{Measurements}

Comparison of different metal shapes (plate, foil, and particle) and sensitivity. To compare the XRF sensitivities when using the micro-XRF (non-confocal) and C-M-XRF, different shaping $\mathrm{Cu}$ samples (plate, foil, and particle) were measured by the nonconfocal 3D micro-XRF and the confocal 3D micro-XRF
Table 1 Chemical composition of mineral sand (JLk-1)

\begin{tabular}{cccc}
\hline Chemical species & Conc., wt $\%$ & Chemical species & Conc., wt $\%$ \\
\hline $\mathrm{Al}_{2} \mathrm{O}_{3}$ & 16.73 & $\mathrm{TiO}_{2}$ & 0.668 \\
$\mathrm{SiO}_{2}$ & 57.16 & $\mathrm{MnO}$ & 0.266 \\
$\mathrm{~K}_{2} \mathrm{O}$ & 2.805 & $\mathrm{Fe}_{2} \mathrm{O}_{3}$ & 6.929 \\
\hline
\end{tabular}

instruments. The X-ray tube was operated at $50 \mathrm{kV}$ and $0.5 \mathrm{~mA}$. The measurement time was set to $200 \mathrm{~s}$, the X-ray beam diameter was focused to $10 \mu \mathrm{m}$, and the working distance was adjusted by $1 \mathrm{~mm}$. All measurements were performed under a vacuum (lower than $10 \mathrm{~Pa}$ ).

Measurements of minute particles of $\mathrm{Fe}, \mathrm{Cu}$, and mineral sand. Several metal particles ( $\mathrm{Fe}, \mathrm{Cu}$, and mineral sand) were set on the acrylic plate with a thickness of $2 \mathrm{~mm}$ using double-sided tape. The $150 \times$ magnified optical images observed with the digital microscope VHX-900 (Manufactured by Keyence Corporation) of the particles are presented in Fig. 3. Particles marked with white line checks in the figures were measured by the non-confocal 3D micro-XRF and the confocal 3D micro$\mathrm{XRF}$ instruments. The measurement conditions were the same as when $\mathrm{Cu}$ plate, foil and particle were measured.

\section{Results and Discussion}

Comparison of different metal shapes (plate, foil, and particle) and sensitivity

The $\mathrm{Cu}$ plate, $\mathrm{Cu}$ foil, and $\mathrm{Cu}$ particle were measured by $\mathrm{C}-\mathrm{M}-\mathrm{XRF}$ and micro-XRF to compare the XRF sensitivities, depending on the shape of the samples, such as the thickness and size. The peak net intensity $\left(I_{\mathrm{Net}}\right)$ and the background intensity $\left(I_{\mathrm{BG}}\right)$ were calculated within the range of the full width at half maximum $(\mathrm{FWHM})$ of the peaks and the $\mathrm{SN}$ ratio $\left(I_{\mathrm{Net}} /\right.$ $I_{\mathrm{BG}}$ ) were obtained.

In the micro-XRF, the polycapillary half lens was just removed, so the distance between the SDD and the sample was about $36 \mathrm{~mm}$. Therefore, it was considered that the effective solid angle of the fluorescent X-rays generated from the sample was lowered, and as a result, the overall intensities were lowered.

We discuss the relative changes in $I_{\mathrm{Net}}$ and $I_{\mathrm{BG}}$, and the SN ratios. A comparison of the intensities of the $\mathrm{Cu}$ plate, $\mathrm{Cu}$ foil, and $\mathrm{Cu}$ particle using $\mathrm{C}-\mathrm{M}-\mathrm{XRF}$ and micro-XRF is presented in 

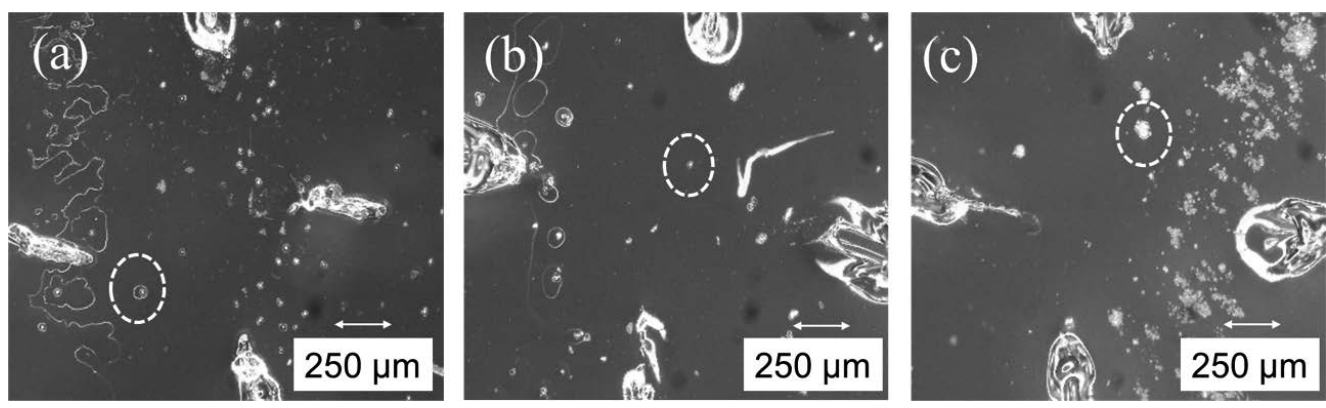

Fig. 3 Optical sample images of (a) Cu particle, (b) Fe particle, and (c) JLk-1 particle, indicated by dotted broken circles (Observed with the digital microscope VHX-800).

(a) $I_{\text {Net }}$

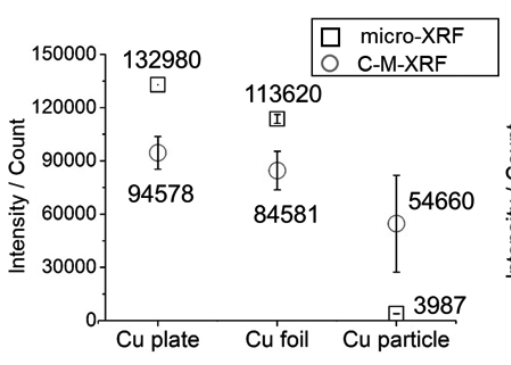

(b) $I_{B G}$

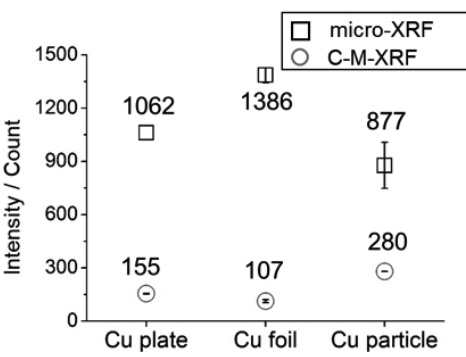

(c) $\mathrm{SN}$ ratio $\left(I_{\mathrm{Net}} / I_{\mathrm{BG}}\right)$

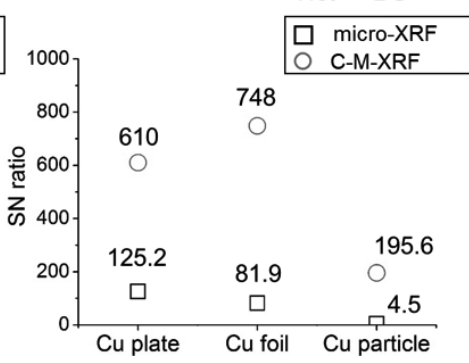

Fig. 4 (a) $I_{\mathrm{Net}}$ and (b) $I_{\mathrm{BG}}$, and (c) SN ratios $\left(I_{\mathrm{Net}} / I_{\mathrm{BG}}\right)$ of Cu plate, foil, and particle. Error bars indicate the range of $\pm \sigma$ value.

Fig. 4. In the micro-XRF, the $I_{\text {Net }}$ of the $\mathrm{Cu}$ particle was much lower than those of the $\mathrm{Cu}$ plate and foil. Under the $\mathrm{C}-\mathrm{M}-\mathrm{XRF}$ arrangement, XRF emitted from the particle was collected with a large solid angle (approximately, 32 degrees) by applying the polycapillary half lens; therefore, the intensity was as large as 54660 counts, as shown in Fig. 4(a). On the other hand, the collecting solid angle in the micro-XRF was quite small (approximately, 11 degrees); therefore, the intensity for $\mathrm{Cu}$ particle was very small, 3987 counts, as shown in Fig. 4(a). Meanwhile, in the C-M-XRF, the $I_{\mathrm{Net}}$ of the Cu plate, foil, and particle were similar. Therefore, the C-M-XRF enabled the $\mathrm{XRF}$ to be obtained selectively from the confocal volume, and it had higher sensitivity for the detection of thin and minute samples.

As shown in Fig. 4(c), the $\mathrm{SN}$ ratios of the $\mathrm{Cu}$ plate, foil and particle obtained by the C-M-XRF were 4.87 (610/125.2), 9.13 (748/81.9), and $43.5(195.6 / 4.5)$ times those of the $I_{\mathrm{BG}}$ obtained by the micro-XRF, respectively. This is because, in the nonconfocal XRF, the primary X-rays were absorbed inside the sample, and more scattered X-rays were detected not only from the surface, but also from the deep region. Since these samples were set on an acrylic plate, the scattered X-rays would be strongly observed in the spectrum. Meanwhile, in the C-M$\mathrm{XRF}$, as the X-rays were detected only from the confocal volume, it is presumed that the scattered X-rays from the peripheral and deep regions were reduced, leading to a high SN ratio, as shown in Fig. 4(c).

According to the above results, it was demonstrated that C-MXRF enabled the XRF to be detected selectively from the confocal volume, and it was found that this effect clearly appeared for measurements of thin or minute samples, particularly for particle samples.

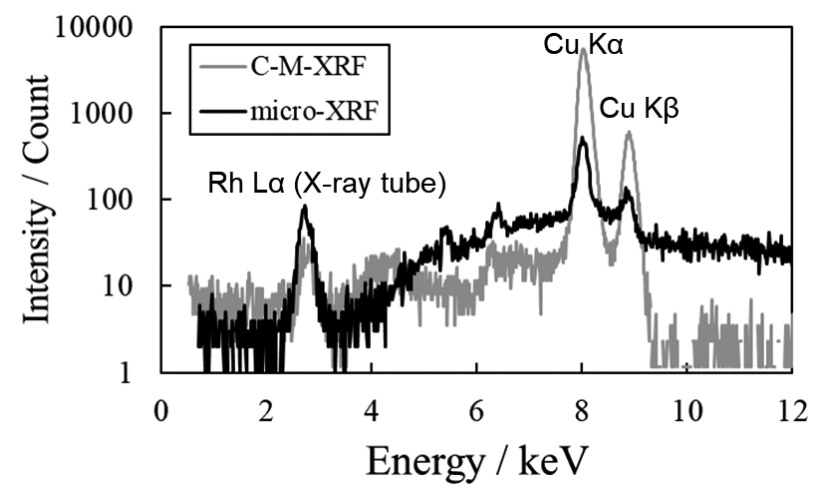

Fig. 5 Comparison of spectra when a $\mathrm{Cu}$ particle is measured by C-M-XRF and micro-XRF.

Measurements of minute particles of $\mathrm{Fe}, \mathrm{Cu}$, and mineral sand In measurements of the particles using by C-M-XRF, to determine the irradiating position more accurately, elemental mapping analysis was performed in the horizontal and vertical directions, and the XRF spectra were obtained at points where the highest XRF intensities were obtained, as shown in Figs. 5 and 6. When measuring the particles using micro-XRF, elemental mapping analysis was performed in the horizontal direction, and the XRF spectrum was obtained at points where the highest XRF intensities were obtained. The spectra comparison of measuring the $\mathrm{Cu}$ and $\mathrm{Fe}$ particles using microXRF and C-M-XRF are shown in log scale in Figs. 5 and 6, respectively. In both figures, the XRF spectra in CM-XRF were observed with very small background, leading to clear characteristic peaks. Figure 7 shows a comparison of spectra 


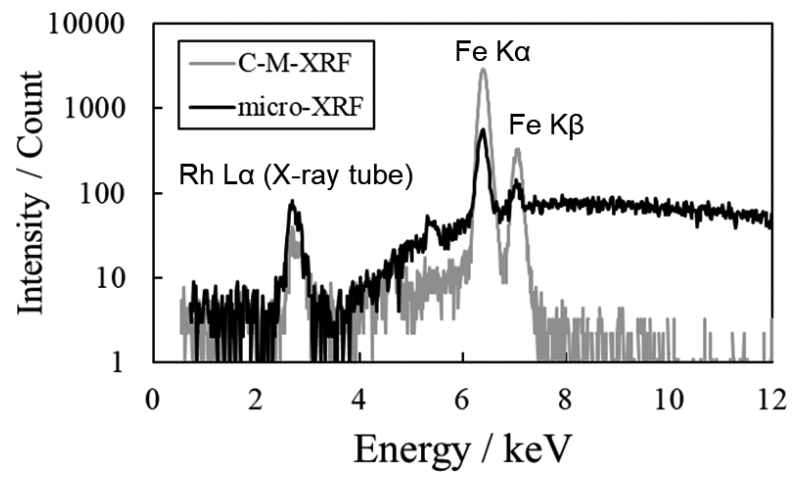

Fig. 6 Comparison of spectra when an Fe particle is measured by $\mathrm{C}-\mathrm{M}-\mathrm{XRF}$ and micro-XRF.

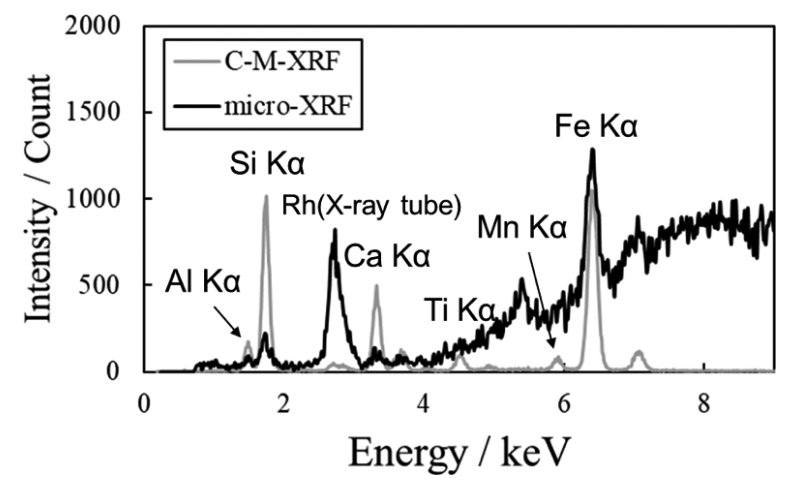

Fig. 7 Comparison of spectra when a mineral particle, JLk-1, is measured by $\mathrm{C}-\mathrm{M}-\mathrm{XRF}$ and micro-XRF.

when mineral particles, JLk-1, were measured by C-M-XRF and micro-XRF. It was found that the $\mathrm{X}$-ray background intensities in micro-XRF were higher than that of C-M-XRF, as shown in Figs, 5, 6 and 7. In the lower energy range (lower approximately $4.0 \mathrm{keV}$ ), the background intensities between the C-M-XRF and micro-XRF were at a similar level, and in the higher energy range (approximately in a range of $4.0-10 \mathrm{keV}$ ), the background intensity levels obtained by the C-M-XRF were lower than those of the micro-XRF. In addition, the reduced $I_{\mathrm{BG}}$ made it possible to detect clear peaks of $\mathrm{Ca} K \alpha$, Ti $K \alpha$ and $\mathrm{Mn} K \alpha$, which were not detected by micro-XRF. It was also confirmed that the intensity of Rh $L \alpha$ obtained by C-M-XRF was lower than micro-XRF, as shown in Fig. 7.

Since the particles were set on the acrylic plate, high scattering $\mathrm{X}$-rays were generated not only from the particles, but also from the acrylic plate. However, the scattered X-rays in the acrylic plates were not detected by C-M-XRF, leading to an extremely low background intensity, as shown in Fig. 7. In addition, when using two polycapillaries in front of the X-ray generator and SDD, the transmission efficiency of high-energy X-rays are not better than that of lower energy range. ${ }^{3}$ Therefore, the $I_{\mathrm{BG}}$ of C-M-XRF was much lower than that of micro-XRF.

The $I_{\mathrm{Net}}$ and $I_{\mathrm{BG}}$ values, as well as the LLD values, of the $\mathrm{Cu}$, $\mathrm{Fe}$, and sand particles measured by micro-XRF and C-M-XRF are summarized in Table 2. The $\mathrm{SN}$ ratio of $\mathrm{Si}$ in the C-M-XRF was approximately 14.6-times that of the micro-XRF, whereas those of the $\mathrm{Fe}$ and $\mathrm{Cu}$ were approximately 21.9 and 43.5-times higher, respectively.

Therefore, it was concluded that the C-M-XRF was effective
Table 2 Net and background intensities $\left(I_{\mathrm{Net}}\right.$ and $\left.I_{\mathrm{BG}}\right)$, SN ratios, and LLD values for $\mathrm{Cu}$ particle, Fe particle, and $\mathrm{Si}$ in JLk-1with a measurement time of $200 \mathrm{~s}$

\begin{tabular}{|c|c|c|c|c|c|c|}
\hline & \multicolumn{2}{|c|}{$\mathrm{Cu}$ particle } & \multicolumn{2}{|c|}{ Fe particle } & \multicolumn{2}{|c|}{$\begin{array}{l}\text { Si in sand } \\
\text { JLk-1 }(27 \%)\end{array}$} \\
\hline & $\begin{array}{c}\text { micro- } \\
\text { XRF }\end{array}$ & $\begin{array}{l}\text { C-M- } \\
\text { XRF }\end{array}$ & $\begin{array}{c}\text { micro- } \\
\text { XRF }\end{array}$ & $\begin{array}{l}\text { C-M- } \\
\text { XRF }\end{array}$ & $\begin{array}{l}\text { micro- } \\
\text { XRF }\end{array}$ & $\begin{array}{l}\text { C-M- } \\
\text { XRF }\end{array}$ \\
\hline$I_{\mathrm{Net}} /$ counts & 3987 & 54660 & 4365 & 28921 & 1087 & 8807 \\
\hline$I_{\mathrm{BG}} /$ counts & 877 & 280 & 506 & 153 & 474 & 262 \\
\hline SN ratio & 4.5 & 195.6 & 8.6 & 189.0 & 2.3 & 33.6 \\
\hline LLD/ng & 0.84 & 0.034 & 0.68 & 0.056 & 4.0 & 0.37 \\
\hline
\end{tabular}

for high-sensitivity measurements of the elements, especially in the high-energy range. In C-M-XRF, since the X-rays are detected only from the confocal volume, the background X-rays were low in the higher energy range.

Evaluation of lower limit of detection (LLD)

Both ends of the range of interest (ROI) of the element peak were approximated by a straight line, the background intensity was subtracted, and the $I_{\mathrm{Net}}$ and $I_{\mathrm{BG}}$ values in the energy range of the FWHM of the peak were calculated and compared. The SN ratio was calculated by dividing $I_{\mathrm{Net}}$ by $I_{\mathrm{BG}}$. The LLD was calculated by

$$
\mathrm{LLD}=3 \times \frac{M}{I_{\mathrm{Net}}} \times \sqrt{\frac{I_{\mathrm{BG}}}{t}} .
$$

In Eq. (1), $M$ indicates the mass of the elements (ng), $t$ indicates the measurement time (s), $I_{\mathrm{Net}}$ indicates the peak intensity (cps), and $I_{\mathrm{BG}}$ indicates the background intensity (cps).

The $I_{\mathrm{Net}}$ and $I_{\mathrm{BG}}$ values, as well as the LLD values of the $\mathrm{Cu}$, $\mathrm{Fe}$, and sand particles measured by micro-XRF and C-M-XRF were summarized in Table 2. The LLD value of the $\mathrm{Cu}$ in the C-M-XRF, are approximately $0.040(0.034 / 0.84)$-times that of micro-XRF, whereas those of the $\mathrm{Fe}$ and $\mathrm{Si}$ were 0.082 $(0.056 / 0.68)$ and $0.093(0.37 / 4.0)$, respectively. It was confirmed that the LLD values in the C-M-XRF were better for all three elements, and that the LLD values were much better for the elements in the higher energy range approximately in the range of $4-10 \mathrm{keV}$.

\section{Conclusions}

In this study, the sensitivities of the XRF of minute metal particles on the plastic substrate using micro-XRF and C-MXRF were compared. First, we examined the effectiveness of the $\mathrm{C}-\mathrm{M}-\mathrm{XRF}$ when analyzing the $\mathrm{Cu}$ plate, foil, and minute particles. The $\mathrm{SN}$ ratio when measuring the minute particle in the C-M-XRF was the highest, at approximately 43.5-times that of the micro-XRF. The superiority of the C-M-XRF was confirmed when the sample was micro particles. Second, we examined the effectiveness of the C-M-XRF when analyzing the $\mathrm{Si}, \mathrm{Fe}$, and $\mathrm{Cu}$ particles. The $\mathrm{C}-\mathrm{M}-\mathrm{XRF}$ improved an $\mathrm{SN}$ ratio that was 14.6 to 43.5 -times higher than that obtained by the micro-XRF. It was confirmed that the C-M-XRF was superior for the analysis of elements with a higher energy range (approximately in the range of $4-10 \mathrm{keV}$ ) e.g. $\mathrm{Cu} \mathrm{K \alpha}$ $(8.04 \mathrm{keV})$. Moreover, the LLD values of the C-M-XRF and micro-XRF were evaluated, and it was confirmed that the C-M$\mathrm{XRF}$ allowed for LLD values that were 10.8 to 24.7-times 
higher than those obtained by the micro-XRF. The detection efficiency of the C-M-XRF was higher for heavy elements, such as $\mathrm{Cu}$, than that of light elements, such as $\mathrm{Si}$.

\section{References}

1. K. H. A. Janssens, F. C. V. Adams, and A. Rindby (ed.), "Microscopic X-ray Fluorescence Analysis", 2000, John Wiley $\&$ Sons Ltd, New York, Chichester, Brisbane, Toronto.

2. B. Beckhoff, B. Kanngießer, N. Langhoff, R. Wedell, and H. Wolff (ed.), "Handbook of Practical X-ray Fluorescence Analysis", 2006, Springer, Berlin Heidelberg.

3. K. Tsuji, J. Injuk, and R.V. Grieken (ed.), "X-Ray Spectrometry: Recent Technological Advances", 2004, John Wiley \& Sons Ltd, Chichester.

4. T. Yonehara, D. Orita, K. Nakano, S. Komatani, S. Ohzawa, A. Bando, H. Uchihara, and K. Tsuji, X-ray Spectrom.,
2010, 39, 78 .

5. M. Ohgaki and K. Shinohara, Adv. X-ray Chem. Anal., 2016, 47, 279.

6. X. Ding, N. Gao, and G. Havrilla, Proc. SPIE, 2000, 4144, 174.

7. B. Kanngießer, W. Malzer, and I. Reiche, Nucl. Instrum. Methods Phys. Res., Sect. B, 2003, 211, 259.

8. Z. Smit, K. Janssens, K. Proost, and I. Langus, Nucl. Instrum. Methods. Phys. Res., Sect. B, 2004, 219-220, 35.

9. B. Kanngieber, W. Malzer, A. F. Rodriguez, and I. Reiche, Spectrochim. Acta, Part B, 2005, 60, 41.

10. L. Vincze, B. Vekemans, F. E. Brenker, G. Falkenberg, K. Rickers, A. Somogyi, M. Kersten, and F. Adams, Anal. Chem., 2004, 76, 6786.

11. B. M. Patterson, J. Campbell, and G. J. Havrilla, X-ray Spectrom., 2010, 39, 184

12. T. Nakazawa and K. Tsuji, X-Ray Spectrom, 2013, $42,374$.

13. R. Yagi and K. Tsuji, X-ray Spectrom., 2015, 44, 186. 